\title{
Innovation and Challenges for the Revision of the Air Quality EU Directive
}

\author{
C. Borrego $^{1,2}$, J. Ginja $^{1}$, C. Ribeiro ${ }^{1}$, M. Coutinho ${ }^{1}$ \\ ${ }^{1}$ Institute of Environment and Development, Campus Universitário, Aveiro, Portugal \\ ${ }^{2}$ CESAM \& Dept. of Environment and Planning, University of Aveiro, Aveiro, Portugal, \\ cborrego@ua.pt
}

\begin{abstract}
The current Directive 2008/50/EC on ambient air quality and cleaner air for Europe establishes the need to reduce pollution to levels that minimise harmful effects on human health, paying particular attention to sensitive populations and the environment as a whole, to improve the monitoring and assessment of air quality including the deposition of pollutants, and to provide information to the public. This work summarises the current status of AQD implementation and identifies new challenges for the future directive revision, namely on the selected pollutants, monitoring strategies and combined use of monitoring and modelling techniques.
\end{abstract}

Key words: Air Quality, Monitoring, Modelling, Reference Methods, Micro-sensors

\section{Introduction}

The current Directive 2008/50/EC [1] on ambient air quality and cleaner air for Europe establishes the need to reduce pollution to levels which minimise harmful effects on human health. Traditionally, the air quality assessment has been based on monitoring data, but due to the sparse spatial coverage of monitoring stations, this assessment has some limitations. In this context modelling techniques appear as relevant tools to improve air quality assessment and studies related to human exposure, providing complete spatial coverage information [2]. Methodologies combining modelling and monitoring data can also contribute to a better air quality forecast, providing improved information to the population and minimizing the occurrence of adverse human health effects. The improved information also allows obtaining a better and broader overview of the air quality to support research and policy makers [3]. This paper presents an overview of current Air Quality Directive (AQD) compliance and identifies new challenges for the future directive revision, namely on the selected pollutants, monitoring strategies and combined use of monitoring and modelling techniques.

\section{Air quality status in Europe}

As a consequence of air pollution policies, since 1990 , emissions of the main air pollutants in Europe have declined, resulting in generally improved air quality across the region. However, in some sectors the reduction was not sufficient in order to meet air quality standards or have even increased emissions of some pollutants. For example, in road transport, emissions of nitrogen oxides $\left(\mathrm{NO}_{\mathrm{x}}\right)$ have not sufficiently decreased to meet air quality standards in many urban areas [9].

The evaluation of the existing air quality standards in the AQD under the EU Clean Air Programme for Europe concludes that they are still insufficient in relation to the WHO Air Quality Guidelines (AQG), which represent the levels where health risks are minimized. Additionally, this analysis indicates that a large proportion of European populations and ecosystems are still exposed to air pollution in exceedances of European and WHO standards as presented in Table 1.

Based on this evaluation, effective air quality policies are essential requiring action and cooperation on global, European, national and local levels. The proposed solution must involve technological development, optimisation of infrastructures and urban planning, and behavioural changes [9]. Nevertheless, the last AQD revision process concluded that further tightening in existing EU air quality standards will be ineffective unless actual cuts in air pollution from the main sources are conducted. In that context, the European Commission (EC) proposed a new Clean Air Policy Package, in late 2013 [4]. This package updates existing legislation controlling harmful emissions from plants and agriculture, with a view to reducing 
their impact on human health and the environment. The package has a number of components, including: (i) a new Clean Air Programme for Europe, with measures to ensure that existing targets are met in the short term, and new air quality objectives for the period up to 2030; (ii) a proposal for a revised National Emissions Ceilings Directive with stricter national emission ceilings for six main pollutants, and provisions for black carbon, which will also help to mitigate climate change; (iii) a proposal, for a new directive to reduce pollution from medium-sized combustion installations of between $1 \mathrm{MW}$ th and $50 \mathrm{MWth}$ (Directive (EU) 2015/2193) [10].

The air policy review indicated that policy should focus on achieving compliance with existing air quality standards by 2020 at the latest, instead of revising air quality reference values, and on using a revised NEC Directive to bring down pollution emissions in the period to 2030.

\section{Innovations and challenges for the Air Quality Directive}

Despite the revision of the AQD in the framework of the EU's Year of Air in 2013, a significant group of questions have been identified allowing the definition of future challenges for the forthcoming air quality policies programs.

New challenges for selected pollutants and air quality monitoring

The current air quality monitoring strategy is manly based on measurements from fixed monitoring stations that are selected to be representative of the exposure of the general population and to comply with the limit values. Under the Review of the Thematic Strategy on Air Pollution, The Network of Air Quality Reference Laboratories (AQUILA) presented recommendations for the revision of the current
AQD [5], including different suggestions, grouped into 10 categories: roles and responsibilities of National Reference Laboratories; inter-comparisons and applying of European Normalisation (EN) standards; approval of measurement devices types; particle measurements and their chemical composition; ozone precursors; mercury and heavy metals; black carbon (BC) / organic carbon (OC); average exposure indicator; deposition of heavy metals and $\mathrm{PAH}$; polycyclic aromatic hydrocarbons (PAH).

Regarding other parameters, the report "Status of black carbon monitoring in ambient air in Europe" [8], also emphasizes the importance of black carbon monitoring, as it allows an integrated approach to complementary environmental policy areas. Black carbon is an example of an air pollutant that affects both human health and is a contributor to climate change. For stations in rural areas, member states have to report the $\mathrm{BC}$ related components on $\mathrm{PM}_{2.5}$ fraction, but the reporting has yet to be fully implemented.

For ozone, recent studies evaluating the reasons for non-compliance of ozone target value set by Directive 2008/50/EC and potential for air quality improvements in relation to ozone pollution propose a more integrated approach for this pollutant and its precursors [7]. It is recommended that assessment of some of the 30 recommended substances $\left(\mathrm{C}_{5}-\mathrm{C}_{9}\right.$ substances) and other species with high ozone formation potential (e.g. formaldehyde) is made mandatory [7].

To address the changes in the observed ozone background concentration trends, improving of monitoring networks, siting criteria and siting density are suggested. Additionally studies with global/hemispheric models are necessary to analyse emission reduction scenarios and study the chemical and physical atmospheric processes [7]. To complement the global simulation, the local scale models are needed.

Tab. 1: Percentage of the urban population in the EU-28 exposed to air pollutant concentrations above EU/WHO values (2011-2013) [9].

\begin{tabular}{|c|c|c|c|c|}
\hline Pollutant & EU reference value & $\begin{array}{c}\text { Exposure } \\
\text { estimate } \%\end{array}$ & WHO AQG & $\begin{array}{c}\text { Exposure } \\
\text { estimate } \%\end{array}$ \\
\hline $\mathrm{PM}_{2.5}$ & $25 \mu \mathrm{g} \cdot \mathrm{m}^{-3}$ (year) & $9-14$ & $10 \mu \mathrm{g} \cdot \mathrm{m}^{-3}$ (year) & $87-93$ \\
\hline $\mathrm{PM}_{10}$ & $50 \mu \mathrm{g} \cdot \mathrm{m}^{-3}$ (day) & $17-30$ & $20 \mu \mathrm{g} \cdot \mathrm{m}^{-3}$ (year) & $61-83$ \\
\hline $\mathrm{O}_{3}$ & $120 \mu \mathrm{g} \cdot \mathrm{m}^{-3}(8-$ hour) & $14-15$ & $100 \mu \mathrm{g} \cdot \mathrm{m}^{-3}(8-\mathrm{hour})$ & $97-98$ \\
\hline $\mathrm{NO}_{2}$ & $40 \mu \mathrm{g} \cdot \mathrm{m}^{-3}$ (year) & $8-12$ & $40 \mu \mathrm{g} \cdot \mathrm{m}^{-3}$ (year) & $8-12$ \\
\hline $\mathrm{BaP}$ & $1 \mathrm{ng} \cdot \mathrm{m}^{-3}$ (year) & $25-28$ & $0.12 \mathrm{ng} \cdot \mathrm{m}^{-3}$ (year) & $85-91$ \\
\hline $\mathrm{SO}_{2}$ & $125 \mu \mathrm{g} \cdot \mathrm{m}^{-3}$ (day) & $<1$ & $20 \mu \mathrm{g} \cdot \mathrm{m}^{-3}$ (day) & $36-37$ \\
\hline
\end{tabular}




\section{New challenges for air quality modelling}

In the scope of the discussion for the new air quality Directive and in order to provide improved assessment, Forum for air quality modelling in Europe (FAIRMODE) has been encouraging to a widest use of modelling techniques to air quality assessment and forecast, as well as the development, validation and application of methodologies combining model results and monitoring data.

In summary, FAIRMODE strongly recommends the use of models for the following applications [6]: (i) assessment of $A Q$ levels to establish the extent of exceedances and establish population exposure; (ii) forecasting $A Q$ levels for short term mitigation and public information and warnings; (iii) source allocation to determine the origin of exceedances and to provide a knowledge basis for planning strategies and also (iv) for the development and assessment of plans and measures to control $A Q$ exceedances.

EU Member States have traditionally used monitoring data to assess $A Q$ to report to the EC. However, monitoring networks present a limited spatial representation to properly evaluate human and ecosystem exposure to concentrations higher than the defined limits, as required by the AQD. Therefore, it seems clear that the way forward to improve $A Q$ assessment concerns the combined use of modelling and monitoring techniques. An example of such a combination is given in Borrego et al. (2015) [2], where these challenges are illustrated by novel monitoring and modelling assessment approaches and innovative exposure assessment methodologies, for which selected examples are provided.

Measures to improve the monitoring networks, siting criteria and siting density

In order to improve the harmonization of air quality assessment across the EU, more information is needed with regard to how Member States are applying the siting criteria. Information on how monitoring stations comply with these criteria is essential to ensure that compliance with limit values is being evaluated in a consistent and harmonized way. In this case an analysis of the representativeness of all measurement sites in Europe is also recommended [7].

Specific for ozone an overall European strategy for measuring ozone precursors and especially VOCs is recommended. Additionally, a higher density of measurement stations of VOC in VOC limited areas, as e.g. in Southern Europe, could be considered. Another suggestion includes formalizing and developing a strategy for number and density of supersites, for ozone and precursors measurements [7].

Moreover, the inclusion of the expertise of the AQUILA and FAIRMODE networks for optimizing the monitoring networks, e.g. for further development and evaluation of air pollution models is recommended [7].

New approaches and new sensing technologies

Low cost sensing technologies for air quality assessment are currently being developed and tested against monitoring reference methods in several initiatives, such as COST Action TD1105 Joint Exercise. The intercomparison of data generated by different microsensor systems with reference analysers will contribute to the assessment of the sensors in a real-world context. In this exercise the overall performance of the sensors suggest that the relevant microsensor platforms, if supported by the proper post processing and data modelling tools, can be used for providing spatially and temporally useful information for air quality levels [1].

Additionally the performance of some commercial sensors has been recently evaluated according to a protocol [15] for lowcost gas sensor evaluation and calibration. In this case, a gas sensor could be accepted as an indicative method if the uncertainty does not exceed the data quality objective. The results confirm that low-cost sensors are a promising technology, with a rapid evolution in the market and performance. The use of low-cost sensors in combination with other tools and sources of information can help to reduce the uncertainty, providing more reliable results, with high spatial and temporal resolution, for general population, air quality managers and policy decisions. The information generated by the widespread diffusion of this new sensing technologies will create both opportunities and challenges for policy-making and science namely in the interoperability of sensors, data quality, security of access and new methods for spatio-temporal analysis [11].

Another class of measurement devices consists of sensor arrays, where recognition software often based on neural networks is used to make the sensor array more specific than the sum of the single sensors. In 2008, a prototype built with metal oxide sensors, after a field neural calibration, was shown to be sensitive and selective for the prediction of benzene in urban environment [14]. In another context supervised learning techniques are used for field calibration methods for low-cost sensors. In this work, for some pollutants, it was found that the best agreement between sensors and reference measurements was observed for supervised 
learning techniques compared to linear and multilinear regression confirm the importance of these techniques for sensor assessment and data treatment [13].

\section{Conclusions}

Air pollution is a complex problem that poses multiple challenges in terms of management and mitigation. Effective action to reduce the impacts of air pollution requires a good understanding of the sources that cause it, as well as up-to-date knowledge of air quality status and its impact on humans and on ecosystems.

The present AQD has introduced a relevant improvement regarding the previous directives, namely opening the possibility of using $A Q$ models in combination with monitoring data for the assessment of air quality in member states. Subsequently the proposed Clean Air Policy Package, in 2013, updated existing legislation, with the focus on controlling emissions and with measures to ensure that existing targets are met in the short term, and new air quality objectives for the period up to 2030 .

Nevertheless, relevant developments to the $A Q D$ have been identified including suggestions on the selected pollutants, monitoring strategies, combined use of monitoring and modelling techniques, and inclusion of new approaches and new sensing technologies.

Methodologies combining the different available tools will produce improved information, to support the decision making process and air quality management.

\section{References}

[1] C. Borrego, A.M. Costa, J. Ginja, M. Amorim, M. Coutinho, K. Karatzas, Th. Sioumis, N. Katsifarakis, K. Konstantinidis, S. De Vito, E. Esposito, P. Smith, N. André, P. Gérard, L.A. Francis, N. Castell, P. Schneider, M. Viana, M.C. Minguillón, W. Reimringer, R.P. Otjes, O. von Sicard, R. Pohle, B. Elen, D. Suriano, V. Pfister, M. Prato, S. Dipinto, M. Penza, Assessment of air quality microsensors versus reference methods: The EuNetAir joint exercise, Atmospheric Environment, Volume 147, December 2016, Pages 246-263, ISSN 1352-2310, http://dx.doi.org/10.1016/j.atmosenv.2016.09.050

[2] C. Borrego, M. Coutinho, A.M. Costa, J. Ginja, C. Ribeiro, A. Monteiro, I. Ribeiro, J. Valente, J.H. Amorim, H. Martins, D. Lopes, A.I. Miranda, Challenges for a New Air Quality Directive: The role of monitoring and modelling techniques, Urban Climate, Volume 14, Part 3, December 2015, Pages 328-341, ISSN 2212-0955, http://dx.doi.org/10.1016/j.uclim.2014.06.007

[3] EC, 2008. Directive 2008/50/EC of the European Parliament and of the Council of 21 May 2008 on ambient air quality and cleaner air for Europe.

[4] EC, 2013. Communication from the Commission to the Council, the European Parliament, the European Economic and Social Committee and the Committee of the Regions - "A Clean Air Programme for Europe", COM (2013) 918 final.

[5] ECORYS, 2013a. Review of: Provisions for Air Quality Measurement, Air Quality Modelling, Management Framework, Assessment, and Public Information; and Stakeholder Consultation Support Assessment of the AQUILA Recommendations for the revision of the current Air Quality Legislation. Final report.

[6] ECORYS, 2013b. Review of: Provisions for Air Quality Measurement, Air Quality Modelling, Management Framework, Assessment, and Public Information; and Stakeholder Consultation Support Assessment of the FAIRMODE Recommendations for the revision of the current Air Quality Legislation. Final report.

[7] ECORYS, 2014. Services to assess the reasons for non-compliance of ozone target value set by Directive 2008/50/EC and potential for air quality improvements in relation to ozone pollution. Final report, DG Environment, January, 2014

[8] EEA, 2013. Status of black carbon monitoring in ambient air in Europe. European Environment Agency. Technical report No 18/2013. ISBN 978-92-9213-415-0; ISSN 1725-2237. http://dx.doi.org/10.2800/10150

[9] EEA, 2015. European Environment Agency, 2015. Air Quality in Europe 2015 Report. EEA Report No 5/2015. EEA, Copenhagen, Denmark, p. 57. http://dx.doi.org/10.2800/62459

[10] EU, 2015. Directive (EU) 2015/2193 of the European Parliament and of the Council of 25 November 2015 on the limitation of emissions of certain pollutants into the air from medium combustion plants.

[11] A. Kotsev, S. Schade, M. Craglia, M. Gerboles, L. Spinelle, M. Signorini, Next Generation Air Quality Platform: Openness and Interoperability for the Internet of Things Sensors. 2016, 16, 403. http://dx.doi.org/10.3390/s16030403

[12] L. Spinelle, M. Gerboles, M. G. Villani, M. Aleixandre, F. Bonavitacola, Field calibration of a cluster of low-cost commercially available sensors for air quality monitoring. Part B: $\mathrm{NO}, \mathrm{CO}$ and $\mathrm{CO}_{2}$, Sensors and Actuators B: Chemical, Volume 238, January 2017 Pages 706-715, ISSN 0925-4005, http://dx.doi.org/10.1016/j.snb.2016.07.036

[13] L. Spinelle, M. Gerboles, M. G: Villani, M. Aleixandre, F. Bonavitacola, Field calibration of a cluster of low-cost available sensors for air quality monitoring. Part $A$ : Ozone and nitrogen dioxide, Sensors and Actuators B: Chemical, Volume 215, August 2015, Pages 249-257, ISSN 0925-4005, http://dx.doi.org/10.1016/j.snb.2015.03.031

[14] S. De Vito, E. Massera, M. Piga, L. Martinotto, G. Di Francia, On field calibration of an electronic nose for benzene estimation in an urban pollution monitoring scenario, Sensors and Actuators B: Chemical, Volume 129, Issue 2, 22 February 2008, Pages 750-757, ISSN 0925-4005, http://dx.doi.org/10.1016/j.snb.2007.09.060

[15] L. Spinelle, M. Gerboles, M. Aleixandre, Protocol of Evaluation and Calibration of Low-Cost Gas Sensors for the Monitoring of Air Pollution; JRC Technical Report; European Commission: Rue Robert Stumper, Luxembourg, 2013. 